\title{
Black hole initial data with a horizon of prescribed intrinsic and extrinsic geometry
}

\author{
Brian Smith
}

\begin{abstract}
The purpose of this work is to construct asymptotically flat, timesymmetric initial data with an apparent horizon of prescribed intrinsic and extrinsic geometry. To do this, we use the parabolic partial differential equation for prescribing scalar curvature. In this equation the horizon geometry is contained within the freely specifiable part of the metric. This contrasts with the conformal method in which the geometry of the horizon can only be specified up to a conformal factor.
\end{abstract}

Asymptotically flat, time-symmetric initial data in general relativity consists of an asymptotically flat Riemannian manifold $(M, g)$ with non-negative scalar curvature $R=R(g) \geq 0$. The interpretation of the scalar curvature is that $R=$ $16 \pi \rho$, where $\rho$ is the local mass density. Within the initial data set, a minimal surface that is outermost with respect to one of the ends of the manifold represents a black hole since it bounds the largest possible set that, according to cosmic censorship, would have to be contained within the black hole region of the Cauchy development of that end. Such a surface is generally referred to as an apparent horizon, and this can only consist of a union of topological spheres [6. In the present work, we shall use this term to indicate one such component.

In the time-symmetric case, black hole initial data shall be here taken to consist of one end of such a manifold external to the region bounded by the apparent horizons. Henceforth, we use the notation $(M, g)$ to indicate this, and so, with the assumption that there is only one horizon, $(M, g)$ is now taken to mean a manifold with apparent horizon boundary $S=\partial M \approx \mathbb{S}^{2}$. This is sufficient for the Cauchy problem since the region interior to the apparent horizons does not affect the Cauchy development exterior to the black hole region of this end. For more on black hole initial data see the references in [11.

2010 Mathematics Subject Classification. 53C21, 53C44, 35K55, 35K57, 35K59, 83C57, $83 \mathrm{C} 05$.

Key words and phrases. scalar curvature, parabolic equations, mean curvature, reactiondiffusion equations, black holes, constraint equations.

This research was funded by the Deutsche Forschungsgemeinschaft project SFB 647 B-4 (Space - Time - Matter. Analytic and Geometric Structures).

(C)0000 (copyright holder) 
Relevant geometric information concerning an apparent horizon consists of its metric $h_{0}$, trace free second fundamental form $\hat{\chi}_{0}$, and the local mass density restricted to the horizon $\rho_{0}=\left.\rho\right|_{S}$. In the present work we shall say that such a horizon is described by $\left(\mathbb{S}^{2}, h_{0}, \hat{\chi}_{0}, \rho_{0}\right)$. Since an apparent horizon must be an outermost minimal surface, it must be area minimizing in that any normal variation of the surface must increase area. By the second variation of area formulaㄹ, this implies that for any non-zero $C^{\infty}$ test function $\varphi$, one must have

$$
\int_{\mathbb{S}^{2}}\left(|\nabla \varphi|_{h_{0}}^{2}+\left(\kappa\left(h_{0}\right)-\frac{1}{2}\left|\hat{\chi}_{0}\right|^{2}-8 \pi \rho_{0}\right) \varphi^{2}\right) \mathrm{d} A_{h_{0}} \geq 0
$$

where $\kappa\left(h_{0}\right)$ is the Gauss curvature of $h_{0}$. We shall be concerned with the slightly stronger situation in which

$$
\int_{\mathbb{S}^{2}}\left(|\nabla \varphi|_{h_{0}}^{2}+\left(\kappa\left(h_{0}\right)-\frac{1}{2}\left|\hat{\chi}_{0}\right|^{2}-8 \pi \rho_{0}\right) \varphi^{2}\right) \mathrm{d} A_{h_{0}}>0
$$

for any non-zero $C^{\infty}$ test function $\varphi$, in which case we shall say that the horizon is strictly stable. Thus, in a sense strict stability is almost necessary. In this work we prove that this condition is sufficient for the existence of asymptotically flat, timesymmetric initial data containing an apparent horizon described by $\left(\mathbb{S}^{2}, h_{0}, \hat{\chi}_{0}, \rho_{0}\right)$. That is, there holds

Main Theorem. Let $h_{0}$ be a Riemannian metric on $\mathbb{S}^{2}$. Let $\hat{\chi}_{0}$ be a symmetric tensorfield of rank $(0,2)$, which is trace free with respect to $h_{0}$. Let $\rho_{0}$ be a nonnegative function. Then there exists asymptotically flat, time-symmetric initial data with a strictly stable horizon boundary described by $\left(\mathbb{S}^{2}, h_{0}, \hat{\chi}_{0}, \rho_{0}\right)$ provided the operator

is negative.

$$
\Delta_{h_{0}}-\left(\kappa\left(h_{0}\right)-\frac{1}{2}\left|\hat{\chi}_{0}\right|^{2}-8 \pi \rho_{0}\right)
$$

As in 11, the main theorem is proved by taking $M=\left[r_{0}, \infty\right) \times \mathbb{S}^{2}$, along with an appropriate local mass density $\rho$, and family of metrics $h(r)$ on $\mathbb{S}^{2}$, and solving for a function $u$ such that

$$
g=u^{2} d r^{2}+h
$$

satisfies $R(g)=16 \pi \rho$. This is accomplished using the parabolic scalar curvature equation 2 , which we use here in the following form:

$$
\tilde{H} \partial_{r} u=u^{2} \Delta_{h} u-(\kappa(h)-8 \pi \rho) u^{3}+\left(\partial_{r} \tilde{H}+\frac{\tilde{H}^{2}+|\tilde{\chi}|^{2}}{2}\right) u,
$$

where $\tilde{\chi}_{A B}=\frac{1}{2} \frac{\partial h_{A B}}{\partial r}$ and $\tilde{H}=h^{A B} \tilde{\chi}_{A B}$. Concerning the foliation spheres $S_{r}=$ $\{r\} \times \mathbb{S}^{2}$, the extrinsic curvature $\chi$ and mean curvature $H$ are related to $\tilde{\chi}$ and $\tilde{H}$ by $\tilde{\chi}=u \chi$ and $\tilde{H}=u H$.

The strength of using the parabolic scalar curvature equation for our purposes is that it allows for the control of the geometry of the foliation $S_{r}$. Indeed, since the mean curvature of this foliation is $H=\tilde{H} / u$, by simply choosing the family $h$ such that $\tilde{H}>0$ for $r>r_{0}$, a minimal surface at $S_{r_{0}}$ must be outermost, and

\footnotetext{
${ }^{1}$ This is actually from the standard second variation formula combined with the Gauss equation. For more on the second variation formula see [5] pp. 169-171.

${ }^{2}$ For more on this equation and its use see $[\mathbf{2},[\mathbf{3},[\mathbf{4},[\mathbf{9}, \mathbf{1 0}, \mathbf{1 3}, \mathbf{1 4}, \mathbf{1 5}$.
} 
hence an apparent horizon. In addition, we can control the intrinsic geometry of $S_{r_{0}}$ simply by the choice of $h\left(r_{0}\right)=h_{0}$. It should be noted that these features of the general method were also of central importance in [1].

The difference between the present work and the previous work lies in how we arrange that $S_{r_{0}}$ is minimal. In 11 this was arranged by requiring that $u \rightarrow \infty$ as $r \rightarrow r_{0}$. Then $S_{r_{0}}$ is, in fact, totally geodesic since the extrinsic curvature of the foliation is given by $\chi=\tilde{\chi} / u$. We cannot use this here since we would now like $\chi$ to be non-trivial at $r_{0}$, and, in fact, prescribed. The only alternative is to arrange that $\tilde{H}$ vanishes initially. Inserting this information into the parabolic scalar curvature equation, we see that the initial data $u_{0}$ is required to satisfy the elliptic equation

$$
\Delta_{h_{0}} u_{0}-\left(\kappa\left(h_{0}\right)-\frac{1}{2}\left|\hat{\chi}_{0}\right|^{2}-8 \pi \rho_{0}\right) u_{0}+\frac{1}{u_{0}}=0,
$$

if, in addition, we assume $\tilde{H}=r-r_{0}$ on a neighborhood of $S_{r_{0}}$.

Thus, the outline of the method in the present work is as follows: We first solve Equation (11) for the initial data $u_{0}$. We then choose the family $h$ to satisfy

$$
\begin{aligned}
\frac{\partial h_{i j}}{\partial r} h^{i j} & >0, \\
\left.\frac{\partial h_{i j}}{\partial r}\right|_{r_{0}} & =2\left(\hat{\chi}_{0}\right)_{i j} u_{0}, \\
\frac{\partial h_{i j}}{\partial r} h^{i j} & =2\left(r-r_{0}\right), r \in\left[r_{0}, r_{0}+\varepsilon\right),
\end{aligned}
$$

and in addition, for $r \geq T, T$ large, we require $h=r^{2} \bar{\gamma}$, where $\bar{\gamma}$ is the standard round metric on $\mathbb{S}^{2}$. We also extend $\rho_{0}$ as a smooth, non-negative, compactly supported function $\rho$ on $M$ in such a way that we may solve the parabolic scalar curvature equation. We then solve the parabolic scalar curvature equation with the initial data $u_{0}$ and check that the resulting function $u$ also has appropriate asymptotic behavior.

Solving the parabolic scalar curvature equation near the horizon cannot, however, be achieved by a simple application of standard parabolic theory. Indeed, the equation will not be parabolic at $r=r_{0}$, and in addition, we would like for $u$ to be $C^{\infty}$ up to and including the initial surface at $r=r_{0}$. We are able to deal with this in the following way: Defining $A=\left(\partial_{r} \tilde{H}+\frac{\tilde{H}^{2}+|\tilde{\chi}|^{2}}{2}\right)$ and $L=\Delta_{h}-(\kappa-8 \pi \rho)$, the parabolic scalar curvature equation takes the form

$$
t \partial_{t} u=u^{2} L u+A u,
$$

where we have defined $t=\left(r-r_{0}\right)$. Let $\tilde{L}$ and $\tilde{A}$ be the $n$-th order Taylor polynomials of $L$ and $A$, respectively. We solve an $n$-th order approximation of the parabolic scalar curvature equation

$$
t \partial_{t} \tilde{u}=\tilde{u}^{2} \tilde{L} \tilde{u}+\tilde{A} \tilde{u}-P,
$$

where $P=a_{0} t^{n+1}+a_{1} t^{n+2}+\cdots+a_{3 n-1} t^{4 n}, a_{0}, a_{1}, \ldots, a_{3 n-1} \in C^{\infty}\left(\mathbb{S}^{2}\right)$. The polynomial $P$ is chosen so that Equation (5) admits a solution of the form

$$
\tilde{u}=u_{0}+u_{1} t+\cdots+u_{n} t^{n},
$$

with $u_{0}, u_{1}, \ldots, u_{n}$ time independent. This is possible since, as will be seen in Section 2 inserting this into (5) yields a sequence of elliptic equations for the $u_{i}$; the first of these is just (1). After having solved the sequence of elliptic equations, 
$P$ is chosen to annihilate the remaining terms in Equation (5). We then insert the decomposition $u=\tilde{u}+v t^{n}$ into the parabolic scalar curvature equation. We are able to solve the resulting equation to obtain a $C^{\infty}$ solution $v$ which satisfies $\left(t \partial_{t}\right)^{m} v \in O(t)$ for $m=0,1, \ldots, n$. The resulting solution $u$ of the parabolic scalar curvature equation has all of the desired properties.

The outline of the paper is as follows: In Section 1 we solve Equation (1) for the function $u_{0}$. In Section 2 we precisely define the family $h$ on a small annular region $A_{\varepsilon}=B_{r_{0}+\varepsilon}(0) \backslash B_{r_{0}}(0)$ about the horizon and, as described in the previous paragraph, we decompose the parabolic scalar curvature equation into Equation (5) and the part for the remainder $v$. In Section 3 we decompose (5) into the sequence of elliptic equations for the $u_{n}$, which are then solved. In Section 4 we solve the equation for the remainder $v$. In the next to the last section we establish a new result for global existence of solutions of the parabolic scalar curvature equation. In the final section the proof of the main theorem is completed by showing how to extend the definition of $h$ smoothly to $\mathbb{R}^{3} \backslash B_{r_{0}+\varepsilon}(0)$ such that the parabolic scalar curvature equation has a unique global solution such that the constructed metric

$$
g=u^{2} d r^{2}+h
$$

is asymptotically flat.

I would like to thank Dr. Kashif Rasul for useful discussions while preparing the manuscript and I would like to thank Professor Robert Bartnik for suggesting prescribing the horizon extrinsic geometry as an interesting problem.

\section{The elliptic equation for the initial data}

This section $\sqrt{3}$ deals with the elliptic equation of the form

$$
\Delta u-a u+\frac{1}{u}=0
$$

on $\left(\mathbb{S}^{2}, h_{0}\right)$, with $a \in C^{\infty}\left(\mathbb{S}^{2}\right)$ and $\Delta=\Delta_{h_{0}}$. The main result of this section is that the equation is solvable provided that the operator $\Delta-a$ is strictly negative. This is a generalization of the following theorem that was proved in [11]:

Theorem 1. Assume $a>0$ and let $a_{*}=\inf _{\mathbb{S}^{2}} a, a^{*}=\sup _{\mathbb{S}^{2}} a$. Then Equation (6) has a positive solution $u \in C^{\infty}$ satisfying $1 / \sqrt{a^{*}} \leq u \leq 1 / \sqrt{a_{*}}$. Within the class of $C^{2}$ functions there are exactly two solutions $\pm u$.

We shall use the method of continuity to generalize this to the case that $\Delta-a$ is merely assumed negative. Namely, we take a positive function $a_{0}$ and define $a_{\tau}=a_{0}(1-\tau)+\tau a$ for $\tau \in[0,1]$ and consider the family of equations

$$
\Delta u_{\tau}-a_{\tau} u_{\tau}+\frac{1}{u_{\tau}}=0 .
$$

By the previous theorem, we know that a solution exists for $\tau=0$. We must only prove that solutions continue to exist with appropriate bounds for all $\tau \in[0,1]$.

Before formally stating and proving the resulting theorem, we note that $a_{\tau}^{*}$ remains positive, we retain the lower bound $u_{\tau} \geq 1 / \sqrt{a_{\tau}^{*}}$, and $\Delta-a_{\tau}$ remains negative. The latter is easily enough checked since the energy

\footnotetext{
${ }^{3}$ This section, as well as Section 3 uses standard methods in elliptic P.D.E theory. For more on these see [7.
} 


$$
E_{\tau}(\varphi)=\int_{\mathbb{S}^{2}}\left(|\nabla \varphi|^{2}+a_{\tau} \varphi^{2}\right) \mathrm{d} A_{h_{0}}
$$

satisfies

$$
E_{\tau}(\varphi)=(1-\tau) E_{0}(\varphi)+\tau E_{1}(\varphi) \geq \delta=\min \left\{\delta_{0}, \delta_{1}\right\}>0,
$$

for all $\varphi \in H^{1}$ with $\|\varphi\|_{L^{2}} \equiv 1$, where $\delta_{i}=\inf \left\{E_{i}(\varphi):\|\varphi\|_{L^{2}} \equiv 1\right\}, i=0,1$; note that each of $\delta_{i}$ has a positive lower bound by the standard argument involving Rellich compactness. By applying $E_{\tau}$ to $\varphi \equiv 1$ the fact that $a_{\tau}^{*}>0$ is easily verified. The proof of $u_{\tau} \geq 1 / \sqrt{a_{\tau}^{*}}$ is a simple application of the maximum principle. Indeed, any solution $u_{\tau} \in C^{2}\left(\mathbb{S}^{2}\right)$ of (17) cannot change signs, and so we may, without loss of generality, assume that $u_{\tau}>0$. At a point $p$ at which the infimum is attained one has $\Delta u_{\tau} \geq 0$; whence at $p$ there holds $a_{\tau} u_{\tau} \geq 1 / u_{\tau}$. Thus, $a_{\tau}(p)>0$ and $\inf u_{\tau}=u_{\tau}(p) \geq 1 / \sqrt{a_{\tau}(p)} \geq 1 / \sqrt{a_{\tau}^{*}}$.

We are now in a position to prove

Theorem 2. Suppose that the operator $\Delta-a$ is negative on $H^{1}$. Then the equation

$$
\Delta u-a u+\frac{1}{u}=0
$$

has a positive $C^{\infty}$ solution that is unique within the class of positive $C^{2}$ functions.

Proof. Let $u_{0}$ denote the solution of Equation (7) at $\tau=0$. The linearization of the associated elliptic operator at $\tau=0$ is just $\Delta-\left(a_{0}+u_{0}^{-2}\right)$, which is bijective as an operator from $C^{2, \alpha}$ into $C^{\alpha}$, for instance. Hence, by the implicit function theorem, classical solutions continue to exist on some interval $\left[0, \tau_{(1)}\right)$. Assume that $\tau_{(1)}$ is maximal in this regard, but $\tau_{(1)}<1$.

In fact, it follows from the negativity of $L_{\tau}=\Delta-a_{\tau}$ that the solutions $u_{\tau}$ are bounded in $L^{2}$ on this interval. To see this, suppose otherwise, and let $\tau_{n}$ be a sequence, $\tau_{n} \rightarrow \tau_{(1)}$ as $n \rightarrow \infty$, such that $\lim _{n \rightarrow \infty}\left\|u_{\tau_{n}}\right\|_{L^{2}}=\infty$. Defining $\tilde{u}_{\tau_{n}}=u_{\tau_{n}} /\left\|u_{\tau_{n}}\right\|_{L^{2}}$, this new sequence of functions satisfies $\left\|\tilde{u}_{\tau_{n}}\right\|_{L^{2}} \equiv 1$. But with $\delta$ as in the paragraph before the theorem, using (7) we can choose $n$ large enough that

$$
E_{\tau_{n}}\left(\tilde{u}_{\tau_{n}}\right)=-\left.\left\langle L_{\tau_{n}} \tilde{u}_{\tau_{n}}, \tilde{u}_{\tau_{n}}\right\rangle\right|_{L^{2}}=\frac{1}{\left\|u_{\tau_{n}}\right\|_{L^{2}}^{2}} \int_{\mathbb{S}^{2}} \mathrm{~d} A_{h_{0}}<\delta,
$$

which is a contradiction.

Using the equation again together with the $L^{2}$ bound on $u_{\tau}$, we obtain a bound

$$
\int_{\mathbb{S}^{2}}\left|\nabla u_{\tau}\right|^{2} \mathrm{~d} A_{h_{0}}=\int_{\mathbb{S}^{2}} a_{\tau} u_{\tau}^{2} \mathrm{~d} A_{h_{0}}-\int_{\mathbb{S}^{2}} \mathrm{~d} A_{h_{0}} \leq C<\infty,
$$

and so $u_{\tau}$ is uniformly bounded in $H^{1}$. Thus, we may use Rellich's compactness theorem to obtain a sequence $u_{\tau_{n}}$ that converges weakly in $H^{1}$ and strongly in $L^{1}$ to an $H^{1}$ function $u_{1}$. Now the $u_{\tau_{n}}$ are essentially bounded from below by a positive constant. This is preserved in the limit, and we have $u_{\tau_{n}}^{-1} \rightarrow u_{1}^{-1}$ in $L^{1}$ as $n \rightarrow \infty$ as well. Hence

$$
\begin{aligned}
0 & =\lim _{n \rightarrow \infty} \int_{\mathbb{S}^{2}}\left(-\nabla u_{\tau_{n}} \cdot \nabla \varphi-a_{\tau_{n}} u_{\tau_{n}} \varphi+\frac{\varphi}{u_{\tau_{n}}}\right) \mathrm{d} A_{h_{0}} \\
& =\int_{\mathbb{S}^{2}}\left(-\nabla u_{1} \cdot \nabla \varphi-a_{1} u_{1} \varphi+\frac{\varphi}{u_{1}}\right) \mathrm{d} A_{h_{0}},
\end{aligned}
$$


for any test function $\varphi \in C^{\infty}\left(\mathbb{S}^{2}\right)$, and thus $u_{1}$ is seen to be an $H^{1}$ weak solution of (7) at $\tau_{(1)}$, which is essentially bounded from below by a positive constant. Standard elliptic regularity theory now shows this to be a $C^{\infty}$ solution.

Applying now the implicit function theorem again as above, we see that there exists a solution on a slightly larger interval $\left[0, \tau_{(1)}+\varepsilon\right)$, in contradiction to the assumption that $\tau_{(1)}$ is maximal. Hence $\tau_{(1)}=1$.

To establish uniqueness within the class of positive $C^{2}$ functions, we consider the difference of two such solutions $u_{1}, u_{2}$. With $\delta u=u_{1}-u_{2}$, one has

$$
\Delta \delta u-\left(a+\frac{1}{u_{1} u_{2}}\right) \delta u=0
$$

But if $\delta u \neq 0$ then $\Delta-a$ cannot be a negative operator, in contradiction to the hypothesis of the theorem.

\section{Decomposition of the parabolic scalar curvature equation near the horizon}

In this section we decompose the parabolic scalar curvature equation on a region near the horizon into the part for the $n$-th order Taylor polynomial

$$
\tilde{u}=u_{0}+u_{1} t+\cdot+u_{n} t^{n}
$$

and the part for the remainder, but first we more precisely define the family $h(t)$.

Using the solution $u_{0}$ of Equation (1), whose existence is guaranteed by the results of last section, and recalling that $t=r-r_{0}$, we define $\tilde{\chi}_{0}=u_{0} \hat{\chi}_{0}$ and take $h_{i j}(t)$ as the solution of the nonlinear ordinary differential equation

$$
h_{i j}^{\prime}=t h_{i j}+2\left(\left(\tilde{\chi}_{0}\right)_{i j}-\frac{1}{2} \operatorname{tr}_{h} \tilde{\chi}_{0} h_{i j}\right) \text {. }
$$

By the standard short time theory for ordinary differential equations, this is possible on some interval $[0, \varepsilon]$, where in addition we may assume that $h$ is $C^{\infty}$. Note that $h$ satisfies Conditions (2) - (4) of the introduction. We extend $\rho_{0}$ to $\left[r_{0}, r_{0}+\varepsilon\right] \times \mathbb{S}^{2}$ by

$$
\rho(t, p)=\rho_{0}(p) .
$$

We may now use the definitions of $h$ and $\rho$ to compute $L=\Delta_{h}-(\kappa(h)-8 \pi \rho)$ and $A=\partial_{r} \tilde{H}+1 / 2\left(\tilde{H}^{2}+|\tilde{\chi}|^{2}\right)$ on $[0, \varepsilon]$. As families of operators from $C^{k+2, \alpha}$ into $C^{k, \alpha}$, we see that $L$ and $A$ are $C^{\infty}$ in time. Whence we have the expansions

$$
\begin{aligned}
& L=L_{0}+L_{1} t+\cdots+L_{n} t^{n}+R_{L} t^{n} \\
& A=A_{0}+A_{1} t+\cdots+A_{n} t^{n}+R_{A} t^{n},
\end{aligned}
$$

where $R_{L}, R_{A}$ verify $\left(t D_{t}\right)^{m} R_{L},\left(t D_{t}\right)^{m} R_{A} \in O(t)$ for $n=0,1, \ldots, n$. Note that $L_{0}=\Delta_{h_{0}}-\left(\kappa\left(h_{0}\right)-8 \pi \rho_{0}\right)$ and $A_{0}=1+\left|\tilde{\chi}_{0}\right|^{2} / 2=1+\left|\hat{\chi}_{0}\right|^{2} u_{0}^{2} / 2$.

To begin to decompose the parabolic scalar curvature equation, define $\tilde{L}=$ $L_{0}+L_{1} t+\cdots+L_{n} t^{n}$ and $\tilde{A}=A_{0}+A_{1} t+\cdots+A_{n} t^{n}$, and assume that $u$ is a solution of the parabolic scalar curvature equation on $[0, \varepsilon]$ that can be expressed as

$$
u=u_{0}+u_{1} t+\cdots+u_{n} t^{n}+v t^{n}
$$

as pointed out in the introduction, $u_{0}$ necessarily has to be the solution of Equation (1). As at the beginning of the section, we take $\tilde{u}=u_{0}+u_{1} t+\cdots+u_{n} t^{n}$. 
Then

$$
\begin{aligned}
u^{2} L u & =u^{2} L \tilde{u}+t^{n} u^{2} L v=\left(\tilde{u}^{2}+2 \tilde{u} v t^{n}+t^{2 n} v^{2}\right) L \tilde{u}+t^{n} u^{2} L v \\
& =\tilde{u}^{2} L \tilde{u}+t^{n} u^{2} L v+\left(2 \tilde{u} L \tilde{u}+t^{n} v L \tilde{u}\right) t^{n} v \\
& =\tilde{u}^{2} \tilde{L} \tilde{u}+t^{n} u^{2} L v+\left(2 \tilde{u} L \tilde{u}+t^{n} v L \tilde{u}\right) t^{n} v+\tilde{u}^{2} t^{n} R_{L} \tilde{u},
\end{aligned}
$$

and

$$
A u=\left(\tilde{A}+t^{n} R_{A}\right)\left(\tilde{u}+t^{n} v\right)=\tilde{A} \tilde{u}+\tilde{A} t^{n} v+t^{2 n} R_{A} v+t^{n} R_{A} \tilde{u} .
$$

Thus, under the above assumptions, the parabolic scalar curvature equation admits the decomposition

$$
\begin{aligned}
& t \frac{\partial \tilde{u}}{\partial t}=\tilde{u}^{2} \tilde{L} \tilde{u}+\tilde{A} \tilde{u}-P \\
& t \frac{\partial v}{\partial t}=u^{2} L v+\left(A+2 \tilde{u} L \tilde{u}+t^{n} v L \tilde{u}-n\right) v+\left(\tilde{u}^{2} R_{L} \tilde{u}+R_{A} \tilde{u}+\frac{P}{t^{n}}\right),
\end{aligned}
$$

where we have introduced an undetermined function $P$. Our procedure shall be to determine $P$ so that we may solve (10) for $\tilde{u}$ an $n$-th order polynomial in time, and then prove the existence of a solution $v$ of (11) satisfying $t^{m} \partial_{t}^{m} \nabla^{k} v \in O(t), m=$ $0,1, \ldots, n, k \in \mathbb{N}$.

\section{A degenerate non-linear parabolic equation with polynomial dependence on time}

In this section we study the degenerate parabolic equation

$$
t \frac{\partial u}{\partial t}=u^{2} L u+A u-P
$$

in the case that the source terms have polynomial expansions in time. More precisely, we assume that

$$
\begin{aligned}
& L=L_{0}+L_{1} t+L_{2} t^{2}+\cdots+L_{n} t^{n} \\
& A=A_{0}+A_{1} t+A_{2} t^{2}+\cdots+A_{n} t^{n},
\end{aligned}
$$

and

$$
P=a_{0} t^{n+1}+a_{1} t^{n+2}+\cdots+a_{3 n-1} t^{4 n},
$$

where $L_{0}=\Delta_{h_{0}}-\left(\kappa\left(h_{0}\right)-8 \pi \rho_{0}\right)$, the $L_{i}$ are second order differential operators on $\mathbb{S}^{2}$ in general, and $a_{i}, A_{i} \in C^{\infty}\left(\mathbb{S}^{2}\right)$ for all $i ; A_{0}$ is here the same as in the last section.

We have the following theorem:

Theorem 3. Assume that $u_{0}$ is a positive $C^{\infty}$ solution of

$$
L_{0} u_{0}+\frac{A_{0}}{u_{0}}=0
$$

and assume furthermore, that $L_{0}$ is a negative operator. Then the $a_{i}$ in the polynomial $P$ may be chosen such that there exists a solution $u$ of Equation (12) of the form

$$
u=u_{0}+u_{1} t+u_{2} t^{2}+\cdots+u_{n} t^{n},
$$

where $u_{1}, u_{2}, \ldots, u_{n}$ are $C^{\infty}$ functions on $\mathbb{S}^{2}$ and $u_{0}$ is as above. 
Proof. We substitute $u=\sum_{i=0}^{n} t^{i} u_{i}, L=\sum_{i=0}^{n} t^{i} L_{i}, A=\sum_{i=0}^{n} t^{i} A_{i}$ into Equation (12) to obtain

$$
\Sigma_{m=0}^{n} m u_{m} t^{m}=\Sigma_{m=0}^{4 n} \Sigma_{a+b+c+d=m} u_{a} u_{b} L_{c} u_{d} t^{m}+\Sigma_{m=0}^{2 n} \Sigma_{a+b=m} A_{a} u_{b} t^{m}-P .
$$

Equating coefficients for the first $n+1$ terms yields the following coupled sequence of equations:

$$
m u_{m}=\Sigma_{a+b+c+d=m} u_{a} u_{b} L_{c} u_{d}+\Sigma_{a+b=m} A_{a} u_{b} .
$$

The first of these is just $u_{0}^{2} L_{0} u_{0}+A_{0} u_{0}=0$, which we have already assumed is uniquely solvable. For $m>0$ we separate the terms involving $u_{m}$ to get

$$
\begin{aligned}
m u_{m}=u_{0}^{2} L_{0} u_{m}+A_{0} u_{m} & +2 u_{0} u_{m} L_{0} u_{0}+\Sigma_{a+b+c+d=m ; a, b, d<m} u_{a} u_{b} L_{c} u_{d} \\
& +\Sigma_{a+b=m, b<m} A_{a} u_{b} .
\end{aligned}
$$

But since $L_{0} u_{0}=-A_{0} / u_{0}$, these can be rewritten as

$$
\begin{gathered}
L_{0} u_{m}-\left(\frac{A_{0}+m}{u_{0}^{2}}\right) u_{m}= \\
-\frac{1}{u_{0}^{2}}\left(\Sigma_{a+b+c+d=m ; a, b, d<m} u_{a} u_{b} L_{c} u_{d}+\Sigma_{a+b=m, b<m} A_{a} u_{b}\right) .
\end{gathered}
$$

Note that the right hand side only contains $u_{i}$ for $i<m$, and so in principle these equations can be solved inductively.

To proceed with the solution, note that when $m=0$ the equation can be solved by hypothesis, and for $m>0$, the equations are linear in $u_{m}$. Furthermore, it was

assumed that $L_{0}$ is negative; whence so is $L_{0}-\frac{\left(A_{0}+m\right)}{u_{0}^{2}}$. Thus, assuming that a unique $C^{k, \alpha}$ solution exists for all $i \leq m$ the right hand side of

$$
\begin{aligned}
& L_{0} u_{m+1}-\left(\frac{A_{0}+(m+1)}{u_{0}^{2}}\right) u_{m+1} \\
= & -\frac{1}{u_{0}^{2}}\left(\Sigma_{a+b+c+d=(m+1) ; a, b, d<(m+1)} u_{a} u_{b} L_{c} u_{d}+\Sigma_{a+b=(m+1), b<(m+1)} A_{a} u_{b}\right)
\end{aligned}
$$

is determined as a $C^{k-2, \alpha}$ function; whence standard elliptic regularity theory shows that there exists a unique solution $u_{m+1} \in C^{k, \alpha}$. Thus, the sequence of $n+1$ equations is uniquely solvable for the functions $u_{1}, u_{2}, \ldots, u_{n}$. Since $k$ was arbitrary, these are in fact $C^{\infty}$.

Now that we have determined the $u_{1}, u_{2}, \ldots, u_{n}$, the proof is completed upon defining

$$
P=\Sigma_{m=n+1}^{4 n} \Sigma_{a+b+c+d=m} u_{a} u_{b} L_{c} u_{d} t^{m}+\Sigma_{m=n+1}^{2 n} \Sigma_{a+b=m} A_{a} u_{b} t^{m} .
$$

\section{The parabolic equation for the remainder}

We now need to prove that there exists a unique solution $v$ of Equation (11) such that $v \rightarrow 0$ as $t \rightarrow 0$. The first step in this direction shall be an $L^{2}$ bound for solutions with small initial data near $t=0$. To do this, we separate the terms in the equation according to their order of growth in $t$. Recall that the equation is

$$
t \frac{\partial v}{\partial t}=u^{2} L v+\left(A+2 \tilde{u} L \tilde{u}+t^{n} v L \tilde{u}-n\right) v+\left(\tilde{u}^{2} R_{L} \tilde{u}+R_{A} \tilde{u}+\frac{P}{t^{n}}\right) .
$$


using the expansions of $A, L$, and $P$, and recalling that $\left(t D_{t}\right)^{m} R_{L},\left(t D_{t}\right)^{m} R_{A} \in$ $O(t), m=0,1,2, \ldots, n$, we write this as

$$
t \frac{\partial v}{\partial t}=u^{2} L v+\left(A_{0}+2 u_{0} L_{0} u_{0}-n+\psi_{1}+v \psi_{2}\right) v+\psi_{3},
$$

where $\psi_{1}, \psi_{2}, \psi_{3}$ are $C^{\infty}$ functions $\psi_{i}: \mathbb{S}^{2} \times \mathbb{R}^{+} \rightarrow \mathbb{R}$ that satisfy $\left(t D_{t}\right)^{m} \psi_{i} \in$ $O(t), m=0,1,2, \ldots, n$ when regarded as functions $\psi_{i}: \mathbb{R}^{+} \rightarrow C^{k}\left(\mathbb{S}^{2}\right)$. But $L_{0} u_{0}=$ $-A / u_{0}$, and so we have

$$
t \frac{\partial v}{\partial t}=u^{2} L v+\left(-A_{0}-n+\psi_{1}+\psi_{2} v\right) v+\psi_{3} .
$$

We would like to prove $L^{2}$ bounds for the solution $v$. To that end, the following lemma is useful:

Lemma 4. Let $L$ be as above, and suppose on $[0, \varepsilon]$ there holds $-\int_{\mathbb{S}^{2}} \varphi L \varphi \mathrm{d} A_{h} \geq$ $\lambda_{0} \int_{\mathbb{S}^{2}} \varphi^{2} \mathrm{~d} A_{h}$ for all $\varphi \in H^{1}\left(\mathbb{S}^{2}\right)$. Let $\psi_{0}: \mathbb{R}^{2} \rightarrow \mathbb{R}, \psi_{0} \in C^{1}$, be such that $\psi_{0}(0,0)=1$. Suppose that $v \in H^{1}\left(\mathbb{S}^{2}\right)$. Then there exists $\delta \leq \varepsilon$ such that if $|t|,|v|<\delta$ then $-\int_{\mathbb{S}^{2}} v \psi_{0}(t, v) L v \mathrm{~d} A_{h} \geq \frac{\lambda_{0}}{2} \int_{\mathbb{S}^{2}} v^{2} \mathrm{~d} A_{h}$.

The proof of this lemma is a fairly straightforward estimate and shall be omitted. The lemma allows us to use techniques for linear equations to derive $L^{2}$ bounds, as contained in the next lemma.

Lemma 5. There exists $\delta, C>0$ such that if $0<t_{1}<\delta$ and $v$ is a solution of Equation (14) on $\left[t_{0}, t_{1}\right]$ with $\left.v\right|_{t_{0}}=0$ and $|v|<\delta$ then $\left\|\left.v\right|_{t}\right\|_{L^{2}\left(\mathbb{S}^{2}\right)} \leq C t$.

Proof. We shall make the change of variables $t=e^{\tau}$ so that Equation (14) becomes

$$
\frac{\partial v}{\partial \tau}=u^{2} L v-\left(A_{0}+n+O\left(e^{\tau}\right)+O\left(e^{\tau}\right) v\right) v+O\left(e^{\tau}\right) .
$$

We define $\tau_{0}=\log t_{0}, \tau_{1}=\log t_{1}$. Multiplying by $v / u_{0}^{2}$ and integrating yields

$$
\frac{1}{2} \frac{d}{d \tau} \int_{\mathbb{S}^{2}} \frac{v^{2}}{u_{0}^{2}} \mathrm{~d} A_{h}-\frac{1}{2} \int_{\mathbb{S}^{2}} \frac{v^{2}}{u_{0}^{2}} \tilde{H} \mathrm{~d} A_{h} \leq \int_{\mathbb{S}^{2}}\left(v \frac{u^{2}}{u_{0}^{2}} L v+\frac{C}{u_{0}^{2}} v e^{\tau}\right) \mathrm{d} A_{h},
$$

provided that we make an initial choice of $\delta$ such that the coefficient of the linear term is negative. Using now the previous lemma with a possibly smaller choice of $\delta$ and using $v e^{\tau} \leq \frac{1}{2}\left(a v^{2}+\frac{1}{a} e^{2 \tau}\right)$, we have

$$
\begin{gathered}
\frac{1}{2} \frac{d}{d \tau} \int_{\mathbb{S}^{2}} \frac{v^{2}}{u_{0}^{2}} \mathrm{~d} A_{h} \leq-\frac{\lambda_{0}}{2} \int_{\mathbb{S}^{2}} v^{2} \mathrm{~d} A_{h}+\frac{e^{\tau}}{2 \min u_{0}^{2}} \int_{\mathbb{S}^{2}} v^{2} \mathrm{~d} A_{h} \\
+\frac{a C}{2 \min u_{0}^{2}} \int_{\mathbb{S}^{2}} v^{2} \mathrm{~d} A_{h}+\frac{C}{2 a \min u_{0}^{2}} \max _{\left[\tau_{0}, \tau_{1}\right]} \operatorname{area}_{h}\left(\mathbb{S}^{2}\right) e^{2 \tau},
\end{gathered}
$$

where we have also used $\tilde{H}=e^{\tau}$. Thus, defining $w=v / u_{0}$, choosing $a$ correctly, and choosing $\delta$ perhaps smaller still, we have

$$
\frac{1}{2} \frac{d}{d \tau} \int_{\mathbb{S}^{2}} w^{2} \mathrm{~d} A_{h} \leq C e^{2 \tau}
$$

Integrating over $\left[\tau_{0}, \tau\right]$ we get

$$
\frac{1}{2} \int_{\mathbb{S}^{2}} w^{2}(\tau, \cdot) \mathrm{d} A_{h}(\tau) \leq C \int_{\tau_{1}}^{\tau} e^{2 \tau^{\prime}} \mathrm{d} \tau^{\prime} \leq C\left(e^{2 \tau}-e^{2 \tau_{0}}\right) \leq C e^{2 \tau} .
$$

This yields the conclusion of the lemma since $t=e^{\tau}$. 
Of course, using Moser iteration, one can derive an analogous supremum bound:

Lemma 6. With the hypotheses of the last lemma, $\delta$ can be chosen such that $|v| \leq C t$ on $\left[t_{0}, t_{1}\right]$.

We may now remove the initial assumption on the smallness of $v$ :

Proposition 7. There exists $C, \varepsilon>0$ such that any solution of Equation (11) on $\left[t_{1}, \varepsilon\right]$ with $\left.v\right|_{t_{1}} \equiv 0$ satisfies $|v| \leq C t$ on $\left[t_{1}, \varepsilon\right]$.

Proof. Let $\delta$ and $C$ be as in the previous lemma. Choose $\varepsilon=\delta / 2 C$. With this choice the conclusion holds. Indeed, suppose otherwise. Since $v=0$ at $t_{1}$, by continuity, it is clear that the inequality must hold on some interval $\left[t_{1}, t^{\prime}\right]$; let this be the maximal interval for which $|v| \leq C t$. But we have at $t^{\prime}$ that $|v|_{t^{\prime}} \leq \delta / 2$, and so it is clear that $|v|<\delta$ for some slightly larger interval $\left[t_{1}, t^{\prime}+\varepsilon^{\prime}\right]$; whence the hypotheses of the previous lemma are fulfilled, which implies that $|v| \leq C t$ on $\left[t_{1}, t^{\prime}+\varepsilon^{\prime}\right]$ as well.

Applying standard regularity theory to the equation for $v$ with time variable $\tau$ we obtain similar bounds for the derivatives of $v$. To state the result, let $\partial_{t}$ denote the derivative in the time direction, and let $\nabla$ denote covariant differentiation along $\mathbb{S}^{2}$. One has

Proposition 8. For $t_{0}>0$, solutions of Equation (11) on $\left[t_{0}, T\right] \times \mathbb{S}^{2}$ are $C^{\infty}$. Moreover, for any integers $k, l$, there exists $\varepsilon$ and $C$ such that any solution of Equation (11) on $\left[t_{1}, \varepsilon\right]$ with $\left.v\right|_{t_{1}} \equiv 0$ satisfies $\left\|\nabla^{k}\left(t \partial_{t}\right)^{l} v\right\|_{C^{0}\left(\mathbb{S}^{2}\right)} \leq C t$ on $\left[t_{0}, \varepsilon\right]$.

Finally, this may be used together with the Ascoli-Arzela theorem to obtain the existence of a solution of Equation (11) with the desired growth properties.

Theorem 9. There exists $\varepsilon>0$ such that Equation (11) has a unique classical solution on $(0, \varepsilon] \times \mathbb{S}^{2}$ in the class $v \in O(t)$. Furthermore, for any integers $k, l$ there exists a constant $C$ such that $\left\|\nabla^{k}\left(t \partial_{t}\right)^{l} v\right\|_{C^{0}\left(\mathbb{S}^{2}\right)} \leq C t$ on $[0, \varepsilon]$.

Proof. Using the equation with $\tau$ instead as the time variable, this corresponds to the existence of a solution on $(-\infty, \log \varepsilon] \times \mathbb{S}^{2}$ that grows like $e^{\tau}$. To obtain this, let $\varepsilon$ be as in the previous proposition, let $\tau_{n}$ be a sequence with $\tau_{n} \rightarrow-\infty$, and let $v_{n}$ be a sequence of solutions on $\left[\tau_{n}, \log \varepsilon\right.$ ) with $\left.v_{n}\right|_{\tau_{n}} \equiv 0$; the latter exist from standard existence theory. Consider now the functions $\omega_{n}=e^{-\tau} v_{n}$. By virtue of the previous proposition, for every integer $k$ these satisfy $\left\|\omega_{n}\right\|_{C^{k}\left(\left[\tau_{n}, \log \varepsilon\right] \times \mathbb{S}^{2}\right)} \leq C$. By using the Ascoli-Arzela theorem, we may extract a subsequence $v_{m}$ that converges in $C^{k}$ on any set $[T, \log \varepsilon] \times \mathbb{S}^{2}, T>-\infty$ to a function $\omega \in C^{k}$. Inserting this sequence into Equation (11) one finds that $v=e^{\tau} \omega$ must be a solution of Equation (11) on $(-\infty, \log \varepsilon] \times \mathbb{S}^{2}$. Furthermore, the bounds on each $v_{n}$ are maintained through the limiting process so that $v$ satisfies the bounds in the conclusion.

To see that the above constructed solution is unique, let $v_{1}, v_{2}$ be two such solutions of class $O(t)$. Then computing the equation for the difference $\delta v=v_{2}-v_{1}$, we arrive at an equation of the form

$$
\frac{\partial \delta v}{\partial \tau}=\tilde{u}_{2}^{2} L \delta v-\left(A_{0}+n-\psi_{1}-\psi_{2}\left(v_{1}+v_{2}\right)-t^{n}\left(\tilde{u}_{1}+\tilde{u}_{2}\right) L v_{1}\right) \delta v,
$$

\footnotetext{
${ }^{4}$ This section, as well as the next one, makes heavy use of standard parabolic techniques; e.g. Moser iteration, Schauder estimates, etc. For more information on these see [8], for instance. Of course, in our case these have been applied on a compact manifold.
} 
where $\tilde{u}_{i}=u=u_{0}+u_{1} t+\cdots+u_{n} t^{n}+v_{i} t^{n}, i=1,2$. But by virtue of the bounds on $v_{1}, v_{2}$, we may assume $\tau$ is small enough that

$$
A_{0}+n-\psi_{1}-\psi_{2}\left(v_{1}+v_{2}\right)-t^{n}\left(\tilde{u}_{1}+\tilde{u}_{2}\right) L v_{1}>c>0 .
$$

Furthermore, with the help of Lemma 4, we may assume that $\tilde{u}_{2}^{2} L$ is strictly negative. Thus, multiplying by $\delta v$ and integrating, we arrive at an inequality of the form

$$
\frac{d}{d \tau}\|\delta v\|_{L^{2}\left(\mathbb{S}^{2}\right)} \leq-c\|\delta v\|_{L^{2}\left(\mathbb{S}^{2}\right)},
$$

from which we get

$$
\left\|\left.\delta v\right|_{\tau}\right\|_{L^{2}\left(\mathbb{S}^{2}\right)} \leq\left.\|\delta v\|_{L^{2}\left(\mathbb{S}^{2}\right)}\right|_{\tau_{0}} e^{-c\left(\tau-\tau_{0}\right)} .
$$

But using the assumed bounds on $v_{1}, v_{2}$ again, for any $\varepsilon^{\prime}>0$, we may assume that $\left\|\left.\delta v\right|_{\tau_{0}}\right\|_{L^{2}\left(\mathbb{S}^{2}\right)}<\varepsilon^{\prime}$ by assuming $\tau_{0}$ is small enough; whence $\left\|\left.\delta v\right|_{\tau}\right\|_{L^{2}\left(\mathbb{S}^{2}\right)} \leq$ $\varepsilon^{\prime} e^{-c\left(\tau-\tau_{0}\right)}$. Then letting $\tau_{0} \rightarrow-\infty$ we obtain that $\left\|\left.\delta v\right|_{\tau}\right\|_{L^{2}\left(\mathbb{S}^{2}\right)}=0$ for any $\tau$.

\section{Global Existence for the Parabolic Scalar Curvature Equation}

In this section it is established that negativity of the operator $L=\Delta_{h}-$ $(\kappa(h)-8 \pi \rho)$ is enough to guarantee global existence results for the parabolic scalar curvature equation in the case of positive bounded initial data. This is contained in Theorem 13 at the end of the section. This result is proved with the help of several intermediate estimates, which are contained in the next two lemmata, and the proposition that follows.

The first estimate concerns the subcritical equation

$$
\tilde{H} \partial_{r} u=u^{2} \Delta u-f u^{3-\alpha}+A u .
$$

One is able to derive bounds for this equation in the case that $f$ is positive on average and $f \in L^{q}\left(\mathbb{S}^{2}\right)$ for appropriate $q$. Specifically, there holds

Lemma 10. Let $q>2$. Suppose that $f \in L^{q}\left(\mathbb{S}^{2}\right)$ and $f_{0}=\int_{\mathbb{S}^{2}} f \mathrm{~d} A_{h}>0$. Then solutions of the subcritical equation, Equation (15), on $\left[r_{1}, r_{2}\right]$ are bounded for any positive initial data. That is, there holds

$$
u \leq C,
$$

where $C$ depends on

$$
\begin{gathered}
\inf _{\left[r_{1}, r_{2}\right]} f_{0}, \sup _{\left[r_{1}, r_{2}\right]}\left\|\left.f\right|_{r}\right\|_{L^{q}\left(\mathbb{S}^{2}\right)}, \inf _{\left[r_{1}, r_{2}\right] \times \mathbb{S}^{2}} \tilde{H}, \\
\sup _{\left[r_{1}, r_{2}\right] \times \mathbb{S}^{2}}|\tilde{H}|, \sup _{\left[r_{1}, r_{2}\right] \times \mathbb{S}^{2}}\left|\partial_{r} \tilde{H}\right|, \sup _{\left[r_{1}, r_{2}\right] \times \mathbb{S}^{2}}|A|,\left\|\left.u\right|_{r_{1}}\right\|_{L^{3}\left(\mathbb{S}^{2}\right)}, \alpha,\left|r_{2}-r_{1}\right|,
\end{gathered}
$$

and of course the family $h$ on $\left[r_{1}, r_{2}\right]$.

Proof. Using Moser iteration one may establish a supremum bound as long as one has a bound on $\|u\|_{L^{p}\left(\left[r_{1}, r_{2}\right] \times \mathbb{S}^{2}\right)}$ for $p>2$. Hence, it suffices for our purposes to establish an $L^{3}$ bound, which we now do. We shall actually obtain a bound on $\left\|\left.u\right|_{r}\right\|_{L^{3}\left(\mathbb{S}^{2}\right)}$ for each $r \in\left[r_{1}, r_{2}\right]$. 
By standard elliptic theory on a compact manifold, one may write $f=f_{0}+\Delta_{h} \omega$. Multiplying the subcritical equation by $u^{2}$ and integrating over $\mathbb{S}^{2}$ we obtain

$$
\begin{aligned}
& \int_{\mathbb{S}^{2}} \frac{\tilde{H}}{3} \partial_{r} u^{3} \mathrm{~d} A_{h}=\int_{\mathbb{S}^{2}}\left(-4 u^{3}|\nabla u|^{2}-\left(f_{0}+\Delta_{h} \omega\right) u^{5-\alpha}+A u^{3}\right) \mathrm{d} A_{h} \\
& =\int_{\mathbb{S}^{2}}\left(-\frac{16}{25}\left|\nabla u^{\frac{5}{2}}\right|^{2}-f_{0} u^{5-\alpha}+(5-\alpha) \nabla \omega \cdot u^{4-\alpha} \nabla u+A u^{3}\right) \mathrm{d} A_{h} .
\end{aligned}
$$

We now estimate the middle term in the integrand:

$$
\begin{gathered}
(5-\alpha) \nabla \omega \cdot u^{4-\alpha} \nabla u=(5-\alpha) u^{\frac{5}{2}-\alpha} \nabla \omega \cdot u^{\frac{3}{2}} \nabla u \\
\leq \frac{(5-\alpha)}{2}\left(u^{5-2 \alpha} \frac{1}{\varepsilon}|\nabla \omega|^{2}+\varepsilon\left(u^{\frac{3}{2}}\right)^{2}|\nabla u|^{2}\right) \\
\quad=\frac{(5-\alpha)}{2}\left(u^{5-2 \alpha} \frac{1}{\varepsilon}|\nabla \omega|^{2}+\frac{4 \varepsilon}{25}\left|\nabla u^{\frac{5}{2}}\right|^{2}\right) .
\end{gathered}
$$

Thus, choosing $\varepsilon=8 /(5-\alpha)$ we obtain

$$
\begin{gathered}
\int_{\mathbb{S}^{2}} \frac{\tilde{H}}{3} \partial_{r} u^{3} \mathrm{~d} A_{h} \leq-\int_{\mathbb{S}^{2}}\left(u^{5-\alpha} f_{0}-\frac{(5-\alpha)^{2}}{16}|\nabla \omega|^{2} u^{5-2 \alpha}\right) \mathrm{d} A_{h}+\int_{\mathbb{S}^{2}} A u^{3} \mathrm{~d} A_{h} \\
=-\int_{\mathbb{S}^{2}} u^{5-2 \alpha}\left(u^{\alpha} f_{0}-\frac{(5-\alpha)^{2}}{16}|\nabla \omega|^{2}\right) \mathrm{d} A_{h}+\int_{\mathbb{S}^{2}} A u^{3} \mathrm{~d} A_{h} .
\end{gathered}
$$

But, the integrand of the first term on the right can only be negative when

$$
u<\left(\frac{|\nabla \omega|^{2}(5-\alpha)^{2}}{16 f_{0}}\right)^{\frac{1}{\alpha}} .
$$

Whence

$$
\int_{\mathbb{S}^{2}} \frac{\tilde{H}}{3} \partial_{r} u^{3} \mathrm{~d} A_{h} \leq \int_{\mathbb{S}^{2}}\left(\frac{25|\nabla \omega|^{2}}{16 f_{0}}\right)^{\frac{5}{\alpha}-1} f_{0} \mathrm{~d} A_{h}+\int_{\mathbb{S}^{2}} A u^{3} \mathrm{~d} A_{h} .
$$

Using now the fact that $|\nabla \omega(r, \cdot)| \leq C\left\|\left.\left(f-f_{0}\right)\right|_{r}\right\|_{L^{q}\left(\mathbb{S}^{2}\right)}$ (see [1]), we obtain

$$
\partial_{r} \int_{\mathbb{S}^{2}} \tilde{H} u^{3} \mathrm{~d} A_{h} \leq C+C \int_{\mathbb{S}^{2}} \tilde{H} u^{3} \mathrm{~d} A_{h},
$$

where $C$ has the dependencies as in the hypothesis of the theorem. Integrating this yields $\int_{\mathbb{S}^{2}} \tilde{H} u^{3} \mathrm{~d} A_{h} \leq C$ on $\left[r_{1}, r_{2}\right]$, which in turn yields the desired bound upon incorporating the lower bound for $\tilde{H}$ into $C: \int_{\mathbb{S}^{2}} u^{3} \mathrm{~d} A_{h} \leq C$ on $\left[r_{1}, r_{2}\right]$.

The supremum bound is then established via Moser iteration as in the remarks at the beginning of the proof.

By the next lemma, given a bound on $\|u\|_{L^{\varepsilon}\left(\mathbb{S}^{2}, h\right)}$, for some $\varepsilon>0$, we can "absorb" a factor of $u^{\alpha}$ into $f$ in order to write the parabolic scalar curvature equation as the subcritical equation with $f$ replaced by $\tilde{f}=f u^{\alpha}$, where it can be arranged that $\tilde{f}$ is positive on average.

Lemma 11. Let $\varepsilon>0$. Suppose that for all $r \in\left[r_{1}, r_{2}\right]$ we have a bound $\|u\|_{L^{\varepsilon}\left(\mathbb{S}^{2}\right)} \leq$ $C, u \geq c>0$. Let $f_{1}>0$ be such that $\int_{\mathbb{S}^{2}} f \mathrm{~d} A_{h} \geq f_{1}$. Then there exists $0<\alpha<$ 
$\varepsilon / 2, \alpha=\alpha\left(c, C, f_{0}, f, \varepsilon\right)$, such that

$$
\int_{\mathbb{S}^{2}} f u^{\alpha} \mathrm{d} A_{h} \geq \frac{1}{2} \int_{\mathbb{S}^{2}} f \mathrm{~d} A_{h}
$$

Furthermore, for $q=\varepsilon / \alpha>2$, one has that $\left\|f u^{\alpha}\right\|_{L^{q}\left(\mathbb{S}^{2}\right)} \leq C^{\prime}$, where $C^{\prime}$ is a constant $C^{\prime}=C^{\prime}\left(c, C, f_{0}, f, \varepsilon\right)$.

Proof. For the time being, we divide the area element $\mathrm{d} A_{h}$ by $\operatorname{area}_{h}\left(\mathbb{S}^{2}\right)$ so that the total measure is $1: \mathrm{d} \mu=\mathrm{d} A_{h} / \operatorname{area}_{h}\left(\mathbb{S}^{2}\right)$. Up until the last line of the proof any integrals are over $\mathbb{S}^{2}$ with respect to $d \mu$; this holds also for any norms. Thus, our bound on $\|u\|_{L^{\varepsilon}\left(\mathbb{S}^{2}\right)}$ becomes $\|u\|_{L^{\varepsilon}} \leq C / A^{\frac{1}{\varepsilon}}$, but since $\varepsilon$ is here fixed, we shall merely rename our constant $C$.

Let $0<\delta<\frac{1}{2} \int f /\|f\|_{L^{1}}$, and let $\alpha<\varepsilon / 2$ be a small positive number to be chosen later. We have

$$
\int f u^{\alpha}=\int f_{+} u^{\alpha}-f_{-} u^{\alpha} \geq c^{\alpha} \int f_{+}-f_{-} u^{\alpha} .
$$

Let now $p$ be chosen such that $\left\|f_{-}\right\|_{L^{p}}<\sqrt{1+\delta}\left\|f_{-}\right\|_{L^{1}}$, and let $p^{\prime}$ be conjugate to $p$. Then using Hölder's inequality

$$
\int f_{-} u^{\alpha} \leq\left\|f_{-}\right\|_{L^{p}}\left\|u^{\alpha}\right\|_{L^{p^{\prime}}} \leq \sqrt{1+\delta}\left\|f_{-}\right\|_{L^{1}}\left(\int u^{\varepsilon \frac{\alpha p^{\prime}}{\varepsilon}}\right)^{1 / p^{\prime}} .
$$

Assuming $\alpha$ to be chosen small enough that $\alpha p^{\prime} / \varepsilon<1$, another application of Hölder's inequality yields

$$
\int f_{-} u^{\alpha} \leq\left\|f_{-}\right\|_{L^{p}}\left\|u^{\alpha}\right\|_{L^{p^{\prime}}} \leq \sqrt{1+\delta}\left\|f_{-}\right\|_{L^{1}}\|u\|_{L^{\varepsilon}}^{\alpha} .
$$

We now further assume $\alpha$ to be chosen such that $\|u\|_{L^{\varepsilon}}^{\alpha}<\sqrt{1+\delta}$, which we may do by virtue of the bound on $\|u\|_{L^{\varepsilon}}$. Making finally the further restriction on $\alpha$ that it be small enough that $c^{\alpha}>1-\delta$, we have

$$
\int f u^{\alpha}>(1-\delta) \int f_{+}-(1+\delta) \int f_{-}=\int f-\delta\|f\|_{L^{1}}>\int f / 2 .
$$

Returning now to the original measure, this inequality continues to hold. The final part of the conclusion follows from Hölder's inequality.

These two lemmata can be combined to obtain a supremum bound on solutions of the parabolic scalar curvature equation in terms of a bound on $\sup _{r}\|u\|_{L^{\varepsilon}\left(\mathbb{S}^{2}, h\right)}$.

Proposition 12. Let the family of metrics $h$ be defined on $\left[r_{1}, r_{2}\right]$ such that $\Lambda^{-1} \leq$ $\tilde{H} \leq \Lambda$ for a positive constant $\Lambda$. Then if $\|u\|_{L^{\varepsilon}\left(\mathbb{S}^{2}\right)}$ is uniformly bounded on $\left[r_{1}, r_{2}\right]$, the supremum of $u$ is also uniformly bounded.

Proof. As remarked, we absorb a factor of $u^{\alpha}$ into $f$ for $\alpha$ as in the previous lemma. That is, we define $\tilde{f}=f u^{\alpha}$, so that the parabolic scalar curvature equation becomes

$$
\tilde{H} \partial_{r} u=u^{2} \Delta u-\tilde{f} u^{3-\alpha}+A u
$$

where $\int \tilde{f}$ is uniformly bounded from below and $\|\tilde{f}\|_{L^{q}\left(\mathbb{S}^{2}\right)}$ is uniformly bounded with $q$ also as in the previous lemma. Thus, applying Lemma 10, the proposition follows.

We are now in a position to prove 
Theorem 13. Let $h$ be a family of metrics on $\mathbb{S}^{2}$ satisfying $h \in C^{\infty}\left(\left[r_{1}, r_{2}\right] \times \mathbb{S}^{2}\right)$. Let $L=\Delta_{h}-(\kappa(h)-8 \pi \rho)$ so that the parabolic scalar curvature equation can be written

$$
\tilde{H} \partial_{r} u=u^{2} L u+A u .
$$

On the interval $\left[r_{1}, r_{2}\right]$ assume that $\tilde{H}>h_{0}>0$. Then for any smooth positive initial data a solution exists on $\left[r_{1}, r_{2}\right]$ provided the operator $L$ is strictly negative.

Proof. By standard parabolic theory, it is enough to establish upper and lower pointwise bounds on $u$. Since the parabolic scalar curvature equation always admits a positive lower bound on any finite interval, we must only prove a supremum bound, which we now do.

For convenience, put $B=\kappa(h)-8 \pi \rho$. For $0<\sigma<1$, multiply the parabolic scalar curvature equation by $u^{-\sigma}$ and integrate to obtain

$\frac{1}{1-\sigma} \int_{\mathbb{S}^{2}} \tilde{H} \partial_{r} u^{1-\sigma} \mathrm{d} A_{h}=-\int_{\mathbb{S}^{2}}\left((2-\sigma) u^{1-\sigma}|\nabla u|^{2}+B u^{3-\sigma}\right) \mathrm{d} A_{h}+\int_{\mathbb{S}^{2}} A u^{1-\sigma} \mathrm{d} A_{h}$.

But this is equivalent to

$$
\begin{gathered}
\frac{1}{1-\sigma} \frac{d}{d r} \int_{\mathbb{S}^{2}} \tilde{H} u^{1-\sigma} \mathrm{d} A_{h}-\frac{1}{1-\sigma} \int_{\mathbb{S}^{2}} \partial_{r} \tilde{H} u^{1-\sigma} \mathrm{d} A_{h}-\frac{1}{(1-\sigma)} \int_{\mathbb{S}^{2}} \tilde{H}^{2} u^{1-\sigma} \mathrm{d} A_{h} \\
=-\int_{\mathbb{S}^{2}}\left(4 \frac{(2-\sigma)}{(3-\sigma)^{2}}\left|\nabla u^{\frac{3-\sigma}{2}}\right|^{2}+B\left(u^{\frac{3-\sigma}{2}}\right)^{2}\right) \mathrm{d} A_{h}+\int_{\mathbb{S}^{2}} A u^{1-\sigma} \mathrm{d} A_{h}
\end{gathered}
$$

Defining $w=u^{\frac{3-\sigma}{2}}$, we have

$$
\begin{aligned}
\frac{1}{1-\sigma} \frac{d}{d r} \int_{\mathbb{S}^{2}} \tilde{H} u^{1-\sigma} \mathrm{d} A_{h} & \leq-\int_{\mathbb{S}^{2}}\left(4 \frac{(2-\sigma)}{(3-\sigma)^{2}}|\nabla w|^{2}+B w^{2}\right) \mathrm{d} A_{h} \\
+C & \int_{\mathbb{S}^{2}} \tilde{H} u^{1-\sigma} \mathrm{d} A_{h} .
\end{aligned}
$$

Now, by choosing $\sigma$ close enough to 1 , the middle term can be made close enough to $\int_{\mathbb{S}^{2}} w L w \mathrm{~d} A_{h}$ that it will be negative. Assuming $\sigma$ to be so chosen, we have

$$
\frac{d}{d r} \int_{\mathbb{S}^{2}} \tilde{H} u^{1-\sigma} \mathrm{d} A_{h} \leq C \int_{\mathbb{S}^{2}} \tilde{H} u^{1-\sigma} \mathrm{d} A_{h}
$$

Integrating this inequality yields a bound on $\|u\|_{L^{1-\sigma}\left(\mathbb{S}^{2}\right)}$ on any finite interval $\left[r_{1}, r_{2}\right]$. Taking $\varepsilon=1-\sigma$ in the previous proposition establishes the result.

\section{Proof of the Main Theorem}

Collecting the results of sections 1-4 establishes:

Proposition 14. Given $\left(\mathbb{S}^{2}, h_{0}, \hat{\chi}, \rho_{0}\right)$ as in the hypothesis of the Main Theorem, there exists $\varepsilon$, functions $u, \rho$ on $\left[r_{0}, r_{0}+\varepsilon\right] \times \mathbb{S}^{2}$ with $\rho>0$, and a family of metrics $h(r), r \in\left[r_{0}, r_{0}+\varepsilon\right]$ such that the metric

$$
g=u^{2} d r^{2}+h
$$

satisfies $R(g)=16 \pi \rho$ and induces $h_{0}, \hat{\chi}$, respectively, as metric and second fundamental form on $S_{r_{0}}=\left\{r_{0}\right\} \times \mathbb{S}^{2}$. Since $\hat{\chi}$ is trace free then $S_{r_{0}}$ is minimal. 
That is to say, we have now constructed the data on the collar region $\left[r_{0}, r_{0}+\varepsilon\right]$, where it should be noted that the operator $L=\Delta_{h}-(\kappa(h)-8 \pi \rho)$ is negative at $r_{0}+\varepsilon$. We now extend $\rho \geq 0, h$ smoothly to an annular region $\left[r_{0}+\varepsilon, T\right]$ such that

$$
\begin{aligned}
\tilde{H} & >0, \\
\left.\rho\right|_{T} & \equiv 0, \\
\left.h\right|_{T} & =c \bar{\gamma},
\end{aligned}
$$

where $c$ is a constant and $\bar{\gamma}$ is round. In addition, we must make the extension in such a way that we may solve the parabolic scalar curvature equation on this region for any smooth positive initial data at $r_{0}+\varepsilon$. By the result of the last section, Theorem [13, we need only ensure that the operator $L$ remains negative. There are, in general, many extensions that preserve the negativity of $L$. To make such an extension is not difficult, but is somewhat lengthy and technical, and we shall not do this here. For a particular example, see the upcoming work [12]. At any rate, with an appropriate extension of $\rho, h$, and taking initial data for $u$ as given by the metric $g$ already constructed at $r_{0}+\varepsilon$, we solve the parabolic scalar curvature equation on $\left[r_{0}+\varepsilon, T\right]$ so that the data is constructed on this region.

At $T$ the local mass density is 0 and the constructed metric has the form

$$
g=u^{2} d r^{2}+c \bar{\gamma}
$$

One may now follow the constructions presented in previous works, e.g. 3], to construct an asymptotically flat extension of $g$ of local mass density 0 , which is such that the foliation of positive mean curvature spheres extends from the sphere at $T$ to $\infty$. This completes the proof of the main theorem.

\section{References}

1. T. Aubin, Some Nonlinear Problems in Riemannian Geometry, Springer-Verlag, New York 1998.

2. R. BARTNIK, Initial data for the Einstein equations in the quasi-spherical gauge, Gravitation and Astronomy Instrument Design and Astrophysical Prospects, edited by D. McLelland and H.A. Bachor, World Scientific, Singapore 1991.

3. R. BARTNIK, Quasi-spherical metrics and prescribed scalar curvature, J. Diff. Geom. 37 No. 1 (1993) 31-71.

4. R. Bartnik, J. Isenberg, The constraint equations, The Einstein Equations and the Large Scale behavior of Gravitational Fields: 50 Years of the Cauchy problem in General Relativity, edited by Piotr Cruściel et.al, Basel: Birkhäuser 1-38, 2004.

5. I. Chavel, Riemannian Geometry - A modern Introduction, Cambridge University Press, New York 2006.

6. S.W. Hawking, The Event Horizon in Black Holes Les Houches Lectures (1973), edited by C. DeWitt, B.S. DeWitt Amsterdam: North Holland 1972.

7. D. Gilbarg And N.S. Trudinger, Elliptic Partial Differential Equations of Second Order, 2nd. Ed., Springer, Berlin 1998.

8. G. Lieberman, Second Order Parabolic Partial Differential Equations, World Scientific, New Jersey 1996.

9. J. Sharples, Local existence of quasispherical space-time initial data, Journal of mathematical Physics 46052501 (2005).

10. Y. Shi And L. TAm, Positive mass theorem and the boundary behaviors of compact manifolds with nonnegative scalar curvature, J. Diff. Geom. 62 No. 1 (2002) 79-125.

11. B. Sмiтh, Black hole initial data with a horizon of prescribed intrinsic geometry, General Relativity and Gravitation 41 (2009) 1013-1024.

12. B. SмiтH, Black hole initial data with a horizon of fully prescribed geometry, In preparation.

13. B. Smith and G. Weinstein, On the connectedness of the space of initial data for the Einstein equations, Electron. Res. Announc. Amer. Math. Soc. 6 (2000) 52-63. 
14. B. Smith And G. Weinstein, Quasi-convex foliations and asymptotically flat metrics of non-negative scalar curvature, Communications in Analysis and Geometry 12 No. 3 (2004) $511-551$.

15. Mu-Tao Wang and Shing-Tung Yau, A generalization of Liu-Yau's quasi-local mass, Commun. Anal. Geom. 15 No. 2 (2007) 249-282.

Freie Universität Berlin, Arnimallee 3, 14195 Berlin, Germany

E-mail address: bsmith@math.fu-berlin.de 